


\title{
El contenido digital en las administraciones locales: condiciones ontológicas y organizativas para su creación y gestión
}

\author{
Digital content in administrations local: ontological and organizational \\ conditions for its creation and manageme
}

Recibido: 05-07-2019 • Aprobado: 11-08-2020 • Página inicial: 93 - Página final: 114

Sergio Jiménez Meroño*

Resumen: el presente artículo pretende argumentar que los contenidos digitales son los elementos más importantes de interacción entre un sitio web y las personas. Para cumplir con esta pretensión, el artículo mostrará: primero, un balance sobre algunos trabajos dedicados a la creación de contenidos digitales de forma local; segundo, la necesidad de diferentes contenidos digitales cuando se habla de los gobiernos locales; tercero, cómo se pueden crear contenidos en estas organizaciones. Así, se concluirá que la creación de contenidos digitales debe ser adaptada para cada gobierno local.

Palabras clave: contenido digital; gobierno local; web; información; creación.
Abstract: This article aims to argue that digital contents are the most important elements for interaction among a website and people. To achieve this claim, the article will show: first, a balance on some previous papers dedicated to the creation of digital content locally; second, the need for different digital content when talking about local governments; third, how content can be created in these organizations. Thus, it will be concluded that the creation of digital content must be adapted for each local government.

Keywords: Digital content; local government; web; information; creation.

JEL: M19

\footnotetext{
* Doctor en Ciencias Políticas y de la Administración de la Universidad Complutense de Madrid - España, diploma en Ciencias Políticas y Sociales por el Institut d'Etudes Politiques de París, Achievement Award en Inteligencia Web por las Universidades de California Irvine y British Columbia. Consultor especializado en Administración y Gobierno electrónico con más de 10 años de experiencia.

sergio@analiticapublica.es

ORCID: https://orcid.org/0000-0001-5213-1203
} 


\section{Conntexto unmones s.}

\section{Conteúdo digital nas administrações locais: condições ontológicas e organizacionais para a sua criação e gestão}

Resumo: este artigo tem como objetivo argumentar que o conteúdo digital é o elemento mais importante na interação entre um site e as pessoas. Para atender a essa pretensão, o artigo apresentará: primeiro, um balanço de algumas obras dedicadas à criação de conteúdo digital localmente; em segundo lugar, a necessidade de diferentes conteúdos digitais ao falar sobre governos locais; terceiro, como o conteúdo pode ser criado nessas organizações. Assim, concluirá-se que a criação de conteúdos digitais deve ser adaptada para cada governo local.

Palavras-chave: conteúdo digital; governo local; web; informação; criação. 


\section{Introducción}

Uno de los mantras del mundo digital es que el contenido es el rey, es decir, lo que hace que las personas usen una web, que aparezca en buscadores, y que su reputación crezca es fruto de la calidad y adecuación del contenido al público. En este contexto, las administraciones públicas se encuentran con un panorama diferente del que tiene el sector público. Por un lado, no tiene que enfrentarse al mercado para asentar su autoridad como creador legítimo de contenido; por otro, los requisitos normativos y las prácticas de comunicación habitual que desarrollan, le dificulta crear contenidos centrados en las personas a las que se dirigen.

El presente artículo hace una recopilación del estado del arte de la creación de contenidos digitales, adaptado a las características específicas de las administraciones locales. Para ello, partiremos de los atributos que definen el contenido digital como principal elemento de interacción entre entornos digitales y personas. Posteriormente, realizaremos una revisión de las necesidades de comunicación de los gobiernos locales como agentes con una triple naturaleza: gobierno, administración y dinamizadores de la vida local, y cómo esto supone la articulación de diferentes tipos y entornos de contenido. Una tercera parte se centrará en los aspectos estructurales que pueden influir en la capacidad de crear contenidos en la organización, de manera que se puedan prever los riesgos y posibles problemas relativos a la creación y gestión cotidiana de contenidos. Finalmente, plantearemos un mecanismo de gestión y gobernanza de contenidos en los entes locales para poder realizar, de esta manera, una creación sostenible y adaptada a cada caso.

\section{El contenido es lo que define la web}

\section{El contenido marca el camino a las personas}

Lo primero que debemos decir es que un sitio web público generalmente alberga mucha información. Existe información sobre su parte corporativa, sus servicios, sus órganos de gobierno, su actividad y demás. Esto obliga, por pura necesidad, a crear un orden en las cosas. Hay que decidir dónde dejamos cada contenido y cómo lo agrupamos.

¿En qué influye esto a las personas que usan una web? En términos generales hay que entender que las personas permanecen en un espacio digital cuando el contenido al que acceden está alineado con sus expectativas, necesidades y 


\section{Contexto Immones s.}

valores, tal y como indican Eisenberg, Eisenberg \& Davis (2002), afectando a las siguientes áreas del cerebro:

- Cortex prefrontal: influye en las emociones, la planificación y la toma de decisiones.

- El área de Broca: descodifica la complejidad del mensaje; cuanto más compleja es la presentación de un mensaje, más difícil es la comprensión.

- Área de Wrenickes: asocia nombres con conceptos u objetos. Si el área de Broca pone en orden las palabras, la de Wrenickes les da significado. El equilibrio entre contenidos claros, sencillos e identificables con elementos distintivos y sorprendentes permite un mensaje más efectivo.

En este sentido, la creación de contenido digital requiere integrar:

- Relevancia.

- Persuasión que permita abordar la decisión o la comprensión de lo que se ofrece desde diferentes enfoques.

- Integración estética.

Pongamos un ejemplo habitual en el sector público: un ayuntamiento que crea una web y que, a la hora de organizar su sitio, se basa en las áreas de gobierno. Esto podría tener cierta lógica. Sin embargo, nos podemos encontrar fácilmente que la información sobre la creación de empresas y ayudas al acristalamiento de viviendas están en el área de economía y desarrollo sostenible. Además, podemos encontrar que pedir un cubo de basura para una comunidad está en el área de urbanismo, que lleva también los Planes de Ordenación. Estos mecanismos de organización (y son sólo dos ejemplos) son muy comunes y afectan a los "no iniciados" en la institución. En términos generales, la incapacidad de las administraciones de clarificar el lenguaje y articular el contenido necesario para los diferentes perfiles de público generan estrés a las personas que acceden a los servicios, tal y como indica el grupo U.S. Governmente, en su estudio Expectatives and challenges: Informing the future of the federal front door (2016), dicho estudio destaca los siguientes problemas:

- Falta de comprensión del contenido.

- Exceso de información ofrecida.

- Falta de transparencia en el seguimiento del proceso.

En términos reales, esto se traduce en que la gente usa más Google que la propia web municipal para encontrar lo que busca. Esto no es necesariamente 
malo, pero limita desde lo que se puede averiguar lo que busca: si se busca la licencia de obras para la reforma (en el área de urbanismo), a lo mejor no se conoce la información de que existan ayudas para mejorar el acristalamiento.

\section{Los términos que usamos traen o expulsan a las personas}

El complemento habitual a la estructura del contenido es la terminología que usamos. Normalmente, cualquiera que se mueve en una web (y la naturaleza de una web es moverse hasta conseguir lo que se busca)lo hace como si fuera un mapa. En este sentido, las palabras y los términos son como el mapa que permite intuir a dónde llegamos.

Por ello, la definición de los términos es crucial para contactar a las personas. No estamos hablando solo de guiar a las personas, sino directamente de echarles del sitio. Volvamos al ejemplo del ayuntamiento. Puede tener un área de juventud, una de cooperación, una de servicios sociales y otra de participación. ¿Dónde debe buscar ayuda una asociación para inserción laboral de jóvenes y migrantes? Posiblemente en los enlaces "ayudas" o "subvenciones". Pero ¿qué puede entonces tener cada uno de estos elementos que hemos dicho antes? ¿memorias de actividad? ¿programas de gobierno? ¿publicaciones? ¿un directorio? Incluso es posible que, si no hay un trabajo de optimización para buscadores (SEO), ni siquiera un buscador pueda ser de mucha utilidad (Solis, 2017).

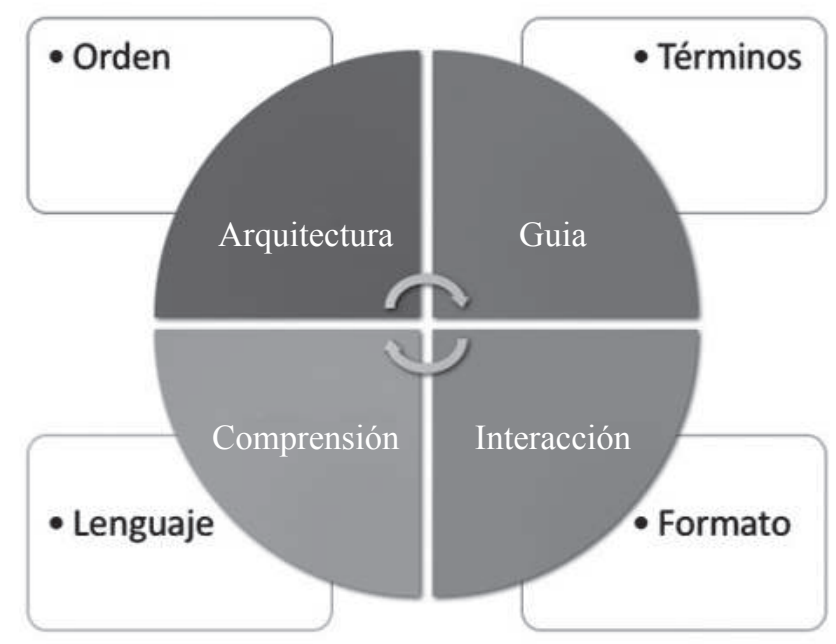

Figura 1. Componentes clave del contenido.

Elaboración propia a partir de Casey (2015). 


\section{Conntexto unmones s.}

La cuestión no es sólo que esto genere confusión, y una tarea de búsqueda no siempre es sencilla, sino que las palabras que usemos orienten, pero también expulsen. Si alguien busca información sobre ayudas a la escolarización y no encuentra una mención en la parte de educación, posiblemente acabe descartando que existan ayudas. Esto es especialmente sensible cuando hablamos de cuestiones que no son de "común conocimiento".

Uno de los problemas del desarrollo de contenidos digitales es su falta de personalización: la ausencia de alineación entre valores, intereses y lenguaje de quien escribe con el que lo lee, genera un abandono prematuro de dicho contenido o un estrés adicional.

El uso de buyer personas en el desarrollo de productos y servicios digitales es un elemento básico para entender la evolución de la economía 2.0. Dicha herramienta parte de la ideación de individuos concretos y definidos que permitan simular y validar el contenido creado para su aprobación. Esto requiere:

- Definir tipos de usuarios o clientes a los que se dirige el servicio.

- Establecer los principales elementos o niveles de interacción e intereses con el servicio, es decir, qué es lo que buscan y qué aspectos le preocupan.

- Establecer aspectos de personalidad que afecten al proceso de la toma de decisiones.

La elaboración de personas se puede realizar de dos maneras, una más empírica y experimental, a través de la realización de entrevistas a perfiles similares (Vaughan, 2015); o bien, como ocurre en las metodologías de design thinking, en un proceso de reflexión de un equipo que conoce al público y que ha realizado un trabajo de empatía previo (Echegaray, Urbano y Barrutieta, 2017).

\section{La integración de estos perfiles en el proceso de diseño y validación del contenido es un elemento medular para crear contenido adecuado en espacios digitales, tal y como lo indica Spool (2008). El lenguaje es como nos entendemos con las personas}

El lenguaje es el tercer nivel. Normalmente, podríamos decir que queremos un lenguaje sencillo, fácil y claro; otros podrían decir que es mejor un lenguaje técnico. En verdad, el lenguaje se tiene que definir en la relación con tu público; es decir, las palabras, los términos, son algo que se dicen a otra(s) persona(s). Por ello, debemos elegir el lenguaje que se adapte al público al que nos dirigimos. 
Por ejemplo, es relativamente fácil encontrar información turística con un lenguaje técnico, geográfico e histórico de alta precisión. Es decir, menciona como atractivo los encantos geológicos del municipio, el valor arquitectónico de la iglesia y los eventos históricos que tienen lugar allí. Esto posiblemente atraiga a gente interesada por la geología, la arquitectura y la historia, pero con conocimientos sobre estos temas. Sin embargo, si se dice que hay un paraje natural único en el mundo con una iglesia medieval en perfecto estado de conservación, el lenguaje supone palabras, términos, y frases específicas para el público al que nos dirigimos.

Hay que identificar a ese público en términos explícitos (por ejemplo, edad, profesión o nivel de estudios), pero también implícitos o internos (estado de ánimo o psicológico). Por ejemplo, es evidente que el perfil del contratante se puede permitir un lenguaje más técnico que la sección de festividades municipales. Sin embargo, el estrés que genera presentar o esperar una resolución a un contrato público es algo que influye igualmente, pero que no es evidente. Quizá el uso del lenguaje y la terminología, así como la construcción de las frases o el diseño del sitio, puede ser la diferencia de pasar cinco o cincuenta segundos hasta encontrar la resolución de este.

\section{El formato dice a qué personas queremos llegar}

Un último elemento es el formato del contenido. El formato afecta del mismo modo al público al que queremos llegar como a las condiciones en que se accede a ese contenido. Pongamos un ejemplo claro: no es lo mismo escribir una página web para mí que para alguien de 26 años o para un pensionado. Hay quien lee todos los detalles (skimming) y quien lee sólo aspectos resaltados rápidamente (scanning). El formato tiene un impacto importante en la creación de un contenido útil, tal y como señala Nielsen (2000). Entre los elementos de formato a considerar, destacan los siguientes:

- Palabras clave.

- Subencabezados explicativos.

- Listados.

- Mantener una idea por párrafo.

Alguien más joven directamente cuando vea un tex to largo, salvo que le interese mucho, se va a volver. Si no sabemos a quién nos dirigimos (y cómo lee el contenido) posiblemente desista de él (Eisenberg \& Eisenber, 2006). El uso de estos elementos permite tanto adaptarse a los lectores de tipo rápido y ocasional sin excluir a los lectores con conocimientos específicos. 


\section{Conntexto unmones s.}

Esto incluye, adicionalmente, algo para lo que el sector público no está preparado del todo: el teléfono móvil. Hoy en día la mayor parte de la navegación en Internet se hace a través de pantallas de unas 5 pulgadas. Un texto de más de 1000 palabras va a tener problemas para ser consumido por el público que usa esencialmente este dispositivo. Especialmente, en determinado canal. En diferentes ayuntamientos, se ha documentado que la duración de sesiones a través de teléfono móvil suele abordar algo menos de un minuto y una sola página, lo que es una diferencia respecto a las sesiones que se realizan en equipos de mesa. Incluso parece indicar que las personas realizan pesquisas previas en el móvil y dejan las tareas más complejas para equipos de mesa. Esto no es necesariamente negativo, solo hay que tenerlo en cuenta a la hora de definir dónde ubicar cada contenido (Casey, 2015).

El contenido atrae o no a determinado público. El contenido en realidad hace que sólo determinado público interactúe a través de la web: configura la realidad del servicio que prestamos. Ahora vamos a ver cómo esa configuración afecta.

\section{El contenido en las web municipales}

\section{Los tres contenidos de la web municipal}

Los gobiernos locales por su naturaleza y tamaño imbrican su naturaleza política y administrativa, esto hace que su relación con la ciudadanía abarque ambas esferas. Es el ayuntamiento donde hay que buscar a los representantes de la ciudadanía, pero también el acceso a los servicios públicos. A esta doble cara hay que añadir una variante más: la promoción o dinamización de actividades que no desarrolla directamente, pero que interesan a la comunidad. De esta manera, esta doble naturaleza se plasma en tres tipos de contenidos (Alba, 1997):

- Información institucional. En ella se presta toda la información sobre la institución política. Esto incluye, además de su composición y normativa, todo lo referente a su gestión, procedimientos y actividad, así como todo lo referente a la participación y transparencia. Es la información política en toda su extensión.

- Servicios públicos. Los servicios públicos incluyen una enorme variedad y grados de profundidad en el mundo digital. Existen servicios de los que solo se puede dar información, otros de los que solo se puede facilitar el acceso y otros que solo se pueden hacer completamente.

- Información municipal. Existe una serie de actividades en las que el ayuntamiento no participa, o lo hace de manera muy residual, pero que 
puede ser de interés para la ciudadanía. Esto abarca ámbitos tan diversos que van desde los negocios locales hasta la liga deportiva del municipio o carreras populares.

Se trata de tres tipos de contenidos diferentes con distintas finalidades para la organización, posibilidades tecnológicas e intereses para la ciudadanía.

\section{El interés de la ciudadanía y la navegación}

La gente no suele buscar información por el origen de esta, sino por sus propias necesidades. Esto es un primer elemento a considerar. Si queremos entender el comportamiento de la ciudadanía tenemos que partir de esto.

\section{Debemos partir de dos cuestiones básicas}

- A las personas le podemos ofrecer un máximo dentro de la web. Es decir, en los sitios de información solo podemos aspirar a que estén al tanto de lo que hay; en la de servicios, avanzar en nuestra tecnología al máximo; y en la de participación, una mayor o menor interacción. Cada bloque de contenido tiene una finalidad, una utilidad para el ciudadano y un objetivo propio (Kaushik, 2007).

- Cada página tiene una función en sí misma. Un sitio web tiene una serie de páginas, cada una de ellas aporta algo a la institución y especialmente a las personas. A través de ellas se orienta a dónde ir, se informa qué se puede hacer o cómo se realizan sus acciones.

Así pues, tenemos dos variables importantes en toda la ecuación: el consumo (lo que las personas usan y cómo lo usan) y el contenido (lo que ofrecemos para que logren lo que necesitan).

\section{Analizando el contenido}

Para proceder al análisis de contenido realizamos las siguientes acciones:

- Catalogando las principales webs. Generalmente un sitio tiene miles de webs, así que lo que hemos hecho es quedarnos en su armazón. Para ello, se revisa el mapa del sitio y cada página referenciada; luego, las catalogamos por la finalidad de ese contenido (si es información/promoción, información corporativa o servicios). 


\section{Conntexto unmones s.}

- Analizando la función de cada web. Hay páginas que sirven para informar a la gente (informacionales); también existen páginas que sirven para que la gente se mueva de un lado a otro, como los menús o índices (navegacionales); finalmente, están las que buscan algún tipo de interacción efectiva (transaccionales) (Muñoz y Elósegui, 2011).

- Estudiando el contenido. Miramos el contenido, no con una revisión exhaustiva, sino para ver qué es lo que hay o lo que falta. Es relativamente fácil encontrar páginas con contenido duplicado o con una sola línea de texto; también las hay con vídeo, fotos, etc. Esto incide posiblemente en el consumo del usuario.

- Evaluando el consumo. Una vez tenemos esta idea, lo que hacemos es obtener unas pocas métricas que nos sirvan para entender el consumo tanto de su temática, naturaleza de su contenido como de su función. Estas métricas nos van a servir para ver si el público consume el contenido como se espera, así como comparar el comportamiento de cada uno de ellos.

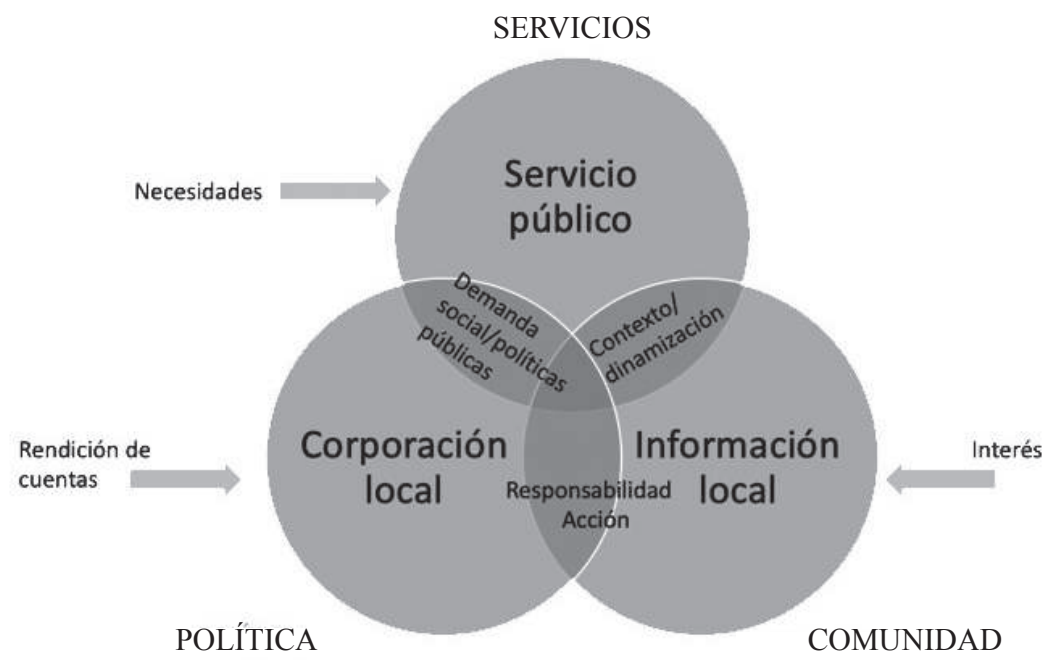

Figura 2. Dimensiones del contenido en una web de un gobierno local.

Elaboración propia.

Sacando conclusiones de los portales municipales

El propósito de la comparación es encontrar:

- Los contenidos que interesan. ¿Qué temas están generando una mayor relevancia en la web? Esto no significa necesariamente que sean los que más guste, sino los que usa más la gente (Kaushik, 2007). 
- Los que funcionan mejor (Casey, 2015). Los contenidos pueden interesar, pero dentro de eso, su naturaleza tiene una finalidad implícita: hay contenidos con mayor fidelización (por ejemplo, espacios de participación) y otros con menos. La idea es prever cómo debería comportarse el público en ese contenido y ver si los datos son fieles o no.

- Encontrar qué bloques tienen problemas en su desarrollo (Casey, 2015). Si encontramos un tema que muestra una excepción en su consumo, debemos prestarle atención. Imaginemos que, por ejemplo, la página para presentar impuestos en un portal tiene muchas más vistas que la de pagarlos. Esto puede indicar que no está bien ubicada en los menús o en sus enlaces. Por otro lado, podemos encontrar que algunas páginas de información tienen un consumo (o número de visitas) muy distinta a otras similares.

La comparación nos sirve para entender mejor dos cosas: lo que el público está buscando y la capacidad de la web de adaptarse a ello.

\section{Planificar los contenidos}

Realmente se trata de pensar cómo reorganizar el contenido. Sin embargo, una reestructuración no es necesariamente algo tan complicado como muchos piensan. Cuando se organiza una cosa así, tenemos cuatro elementos a manejar:

- La ubicación física del contenido. Es decir, en qué espacio está. Esto suele vincularse a la arquitectura de la web, tanto en términos técnicos como en términos organizativos.

- Los menús. Normalmente la gente se maneja a través de menús, más aún, cuando la materia que tienen que gestionar no es una que domine especialmente. Organizar el contenido tiene mucho más que ver con la organización de estos menús y enlaces que facilitan la manera de llegar a la gente de un punto a otro.

- El lenguaje. Los menús son el cómo llegar a un sitio, pero el lenguaje es el qué.

- Las mecánicas de navegación. Hay algunas cosas que no entran directamente en el trabajo, pero que son importantes. Si, por ejemplo, tenemos un sitio de un centro médico con un listado de doctores y hay que entrar en una página personal de cada uno de ellos, acabamos obligando a la gente a ir adelante y atrás. En este caso, puede ser interesante explorar diferentes soluciones tecnológicas para ofrecer el mismo contenido sin tanto movimiento. 


\section{Contexto Imonomes.}

\section{Cada municipio tiene su propio modelo}

Estos elementos deben facilitar la tracción a lo largo de los diferentes bloques en función de los intereses de la ciudadanía. Es decir, lo lógico es que una persona que llegue buscando información sobre cómo montar un negocio, tenga, de una manera $u$ otra, pistas para encontrar información sobre la política local, el concejal, los servicios similares y el comercio.

Cada municipio tiene, como es lógico, su enfoque de qué temas le interesan y cómo gestionarlos. Cada municipio es una comunidad política con sus ideas, sus necesidades, y sus expectativas. La función de una web es adaptarse a esas condiciones y la mejor manera de hacerlo es tratar de entenderlas.

\section{La creación de contenido en las organizaciones públicas}

Los problemas de contenido de una web nunca son fruto de una sola cosa o de una mala decisión. Se trata más bien de una serie de características que asume la organización, algunas de ellas (muy pocas) voluntariamente, otras por la propia evolución de su dinámica. Los grandes problemas de contenido no son culpa de nadie, sino del ciclo vital de las organizaciones y los cambios que experimentan a través de él. Casi como una consecuencia involuntaria. Hay tres dimensiones fundamentales:

- Estructurales.

- Organizativas.

- Productivas.

\section{Anatomía del contenido digital: factores estructurales}

El primer bloque es el que podemos llamar estructural y corresponde a los recursos que tiene la organización. Los sitúo en el primer lugar, porque de todos los que vamos a tratar están profundamente imbuidos en la organización. Es decir, es realmente muy complicado deshacerse o modificar estos elementos, por su importancia y permanencia. Esto no significa que no se pueda "trabajar con ellos", sino que podemos actuar, pero es complicado cambiarlos o deshacerse de ellos. Estos son:

- Personal de la organización. ¿Cuántas personas trabajan en la organización? Un número mayor o menor influye mucho en el contenido. No hablemos tanto del número de personas, sino de la riqueza de puestos y funciones; cuanto más grande y diversa es una organización, más cantidad de 
"autores" de contenido habrá. El número de personas influye mucho en cómo podemos generar contenido, podemos hacer más o menos, pero controlarlo es otra historia.

- Recursos tecnológicos. No vamos a hablar de que la tecnología sea un "matrimonio de por vida", pero sí suele ser una relación a largo plazo. Es decir, cuando se elige o se crea un gestor de contenido y se configura, se está definiendo quién puede hacer qué, y qué recursos se tienen para hacerlo. Cuanto más abierto es el esquema tecnológico, más gente puede generar contenido; cuanto más estrecho o restrictivo, más centralizada será la creación de contenidos. Habrá menos manos para escribir.

\section{Factores organizativos}

Los factores organizativos son los que dependen directamente de la configuración de poder y de recursos de la organización. Es decir, están implicados en la organización, pero, a diferencia de los anteriores, corresponden a decisiones y políticas de esta. Esto significa que, en realidad, sí podemos cambiar (aunque con dificultad) estos elementos. Entre ellos tenemos:

- Estructura organizativa. ¿Estamos en un modelo muy centralizado o poco centralizado? Generalmente, cuando una organización tiene una estructura centralizada, también su gestión de contenidos lo es. En todo caso, hablamos de la creación de contenidos y su aprobación. ¿Puede escribir todo el mundo? Incluso si lo hace, ¿hay una o unas cuantas personas que aprueban, validan o modifican el contenido?

- Enfoque de la generación del contenido. ¿Cuál es el papel de la especialización en el contenido que creamos? Es decir, ¿quién escribe, por ejemplo, de servicios educativos? ¿educación, comunicación o atención al ciudadano? La especialización tiene algo que resaltar: la precisión técnica; aunque esta suele tener una limitación a la hora de hacerse entender. Por otro lado, un área con conocimiento de atención al público puede fallar en la corrección de ese contenido. Normalmente, igual que en el caso anterior, suele haber una relación estrecha, cuanto más centralizada está una organización, menos especializada está la creación de contenidos, y al revés. Esto se debe a que la generación de contenido no es un proceso diferenciado de otros que tienen lugar en la organización. La tensión habitual en las cuestiones de eficiencia y control que plantea la teoría de la agencia es tan aplicable a la creación de contenidos digitales como a la atención al público, así como a gran parte de los negocios actuales tal y como señalan Bendickson, Muldoon, Liguori \& Davis (2016) en los negocios estratégicos. 


\section{Contexto Immones s.}

Un modelo descentralizado genera normalmente una mayor autonomía y proximidad, como apuntan las cuestiones tradicionales de la gestión pública, pero limita el control de los agentes centrales de la organización. Es decir, se pueden generar más contenidos especializados, pero el control por parte de los responsables centrales de la organización es más débil. Por otro lado, en los casos en los que hay un control más centralizado en la creación o aprobación del contenido, como la capacidad de generarlo específico o especializado, se limita. Por ejemplo, cuando un ayuntamiento deja en manos de cada área la publicación de su contenido, esta puede especializarse enormemente en sus mensajes y naturaleza. Sin embargo, esto es más difícilmente supervisable por el gabinete de comunicación, que carece posiblemente de elementos de juicio para saber la pertinencia de determinados elementos concernientes, por ejemplo, a la admisión de niños y niñas en las escuelas infantiles.

Este gabinete puede decidir centrar la creación del contenido, bien sea por el control previo a la publicación que puede eliminar aspectos importantes de información o por la creación directa, lo que da lugar a contenido poco especializado e inconcreto. Estos dos factores están muy marcados por los estructurales. Una administración con muchos empleados y con un sistema relativamente abierto, tiene problemas para un control centralizado. Del mismo modo, una organización con poco personal o recursos o con soluciones técnicas restrictivas suele estar muy centralizado y tener contenido poco especializado.

\section{Factores productivos}

La última dimensión es la que tiene que ver con la cantidad de contenido que producimos. Evidentemente, la producción del contenido llega "al final de la película". Se genera contenido si se tiene gente, temas y tecnología para hacerlo, pero a la vez es el margen donde se tiene más capacidad de decisión y, posiblemente, efectos más limitados. Los dos aspectos a considerar son:

- La cantidad de contenido. ¿Tenemos mucho contenido? La cantidad de contenido es un elemento crucial para hacerlo llegar al público. Cuanto más contenido, más necesidad de ordenarlo, impulsarlo y clarificarlo para que llegue al público.

- La actualización del contenido. La segunda parte es la variación y creación temporal del contenido. El contenido requiere, como mínimo, un mantenimiento: tenerlo al día, informar sus modificaciones, su vigencia o su situación. Además, hay contenidos que son dinámicos y eventuales, tales como noticias, eventos y demás. 
La cantidad y la actualización del contenido son la punta del iceberg que puede apreciar el público. Cuando alguien entra en una web o sigue un perfil social, ve si hay mucho, poco, si se pueden encontrar cosas, si es correcto, o si está actualizado. Sin embargo, la cantidad y actualización depende de los factores anteriores, cuanto más centralizado y restringido es un sistema, menos contenido y menos actualizado suele estar. Del mismo modo, un entorno muy abierto y dinámico puede generar mucho contenido difícilmente gobernable.

\section{Dinámicas de la creación de contenidos}

La evolución del contenido que se crea corresponde más a las dinámicas internas que a la decisión puntual de los responsables públicos. Esto no significa que no podamos hacer nada.

La cuestión es que una vez que sabemos qué es lo que tenemos, podemos hacernos una idea de qué es lo que va mal y qué podemos hacer para mejorar. Esto no suele ser rápido ni sencillo, pero es una manera de recuperar esa voz de la que hablaba.

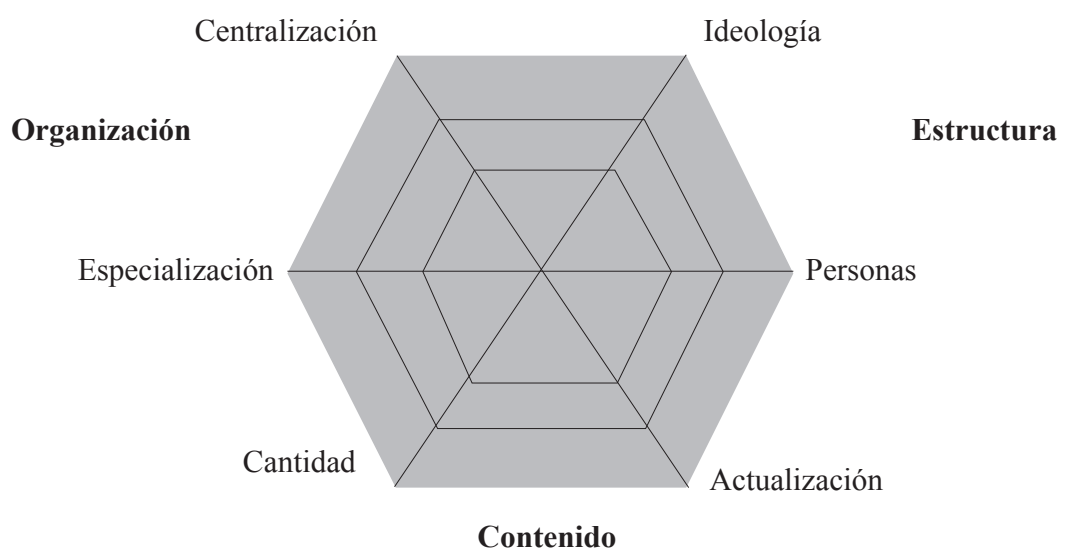

Figura 3. Categorías o extremos de la gestión organizativa del contenido.

Elaboración propia.

Si la planificación del contenido es lo que define qué debemos decir en nuestros medios digitales, la realidad del día a día la desborda. O sea, que si queremos que nuestros contenidos sigan la estrategia marcada necesitamos un plan de gobernanza. 


\section{Contexto Immones s.}

Construir contenido requiere personas que sepan lo que tienen qué hacer y medios para que puedan hacerlo. Ahora vamos a explicar cómo mantener la gobernanza del contenido.

\section{La gobernanza del contenido en la web local}

Los responsables de la gobernanza de contenidos son las personas encargadas de que se mantenga la estrategia planificada. Para ello no basta con sentarlos en un sitio y darles un cartel, sino que necesitan tener unas características y conocimientos determinados.

En primer lugar, son personas que deben tener la posición adecuada en la organización. Esto significa que deben tener autoridad suficiente para hacer cumplir el plan en la misma. Sin embargo, debemos entender que la autoridad requiere no sólo atribuciones de mando, sino tiempo y conocimiento para ejercerla. Es decir, no podemos poner como máximo responsable del contenido a alguien que, o bien no tiene tiempo para supervisar o, que directamente, no está muy interesado.

En segundo lugar, deben entender la estrategia de contenido. Una estrategia de contenido tiene una vida relativamente larga (uno o dos años, fácilmente) y eso supone cambios, imprevistos y sorpresas. Si esas personas no entienden de manera clara y profunda lo que pretende la estrategia, su capacidad de adaptación al cambio será mínima.

Con estas dos condiciones, tenemos tres roles básicos, los cuales se expondrán a continuación.

\section{Responsable de estrategia de contenidos}

Es el principal responsable del contenido de la organización. Una vez que hay una estrategia del contenido, el propio contenido es la estrategia. Los límites de la estrategia son los que deben marcar los del contenido. Esta persona debe estar en un puesto lo bastante elevado en la organización para garantizar el cumplimiento de sus objetivos. Su posición no requiere una carga de trabajo continua, pero sí bastante importante, por lo que debería ser un perfil senior. Entre sus funciones están:

- Liderar la estrategia, impulsando y adaptando los objetivos de esta a los de la organización. 
- Configurar el presupuesto disponible para esta función. Esto incluye no sólo aspectos monetarios, sino también de personal, medios, etc. Debe poder asignar y asegurar el personal y los recursos a la creación de contenidos.

- Ayudar a concretar y adaptar la hoja de ruta de contenidos y comunicar los cambios.

- Comprobar la disponibilidad de herramientas, recursos e instrucciones para garantizar el desarrollo de la estrategia.

- Definir las métricas de éxito.

- Defender las necesidades del contenido frente a otros responsables de la organización, como el de diseño web, operaciones o similares, en cuestiones que generen fricción.

Como señalábamos son perfiles senior. No necesitan una permanencia temporal, pero sí una alta eficacia en el tiempo que dediquen a estas tareas.

\section{Responsable operativo de contenidos}

Es la persona que garantiza y supervisa desde cerca el desarrollo de la estrategia. Es, por lo tanto, quien tiene que gestionar que el contenido se adapte en los términos y condiciones definidos en la estrategia a lo largo de la organización. Para ello debe realizar las siguientes tareas:

- Gestionar la creación del contenido. Esto incluye tanto el contenido que se genera porque estaba planificado, como el que se hace a petición de terceros. Por ejemplo, si hay un evento turístico nuevo en el municipio, su comunicación debería adaptarse a la estrategia de contenido (en tono, forma, objetivo, etc.). Esto hace que deba, por un lado, acoger la petición, adaptarla a las líneas de la organización y habilitar los recursos para hacerlo.

- Gestionar, planificar y supervisar el contenido cotidiano. Evidentemente no se trata de hacer un examen hasta la última coma, pero debe estar al tanto de lo que se publica y evitar desviaciones sustanciales de la estrategia, incluidas las problemáticas.

- Gestionar los ritmos de publicación (cuándo, cómo, quién, en qué medios). Esto es importante, porque en muchas ocasiones los responsables de distintas áreas necesitarán publicaciones que se requieran para crecer a un ritmo preciso, pero sin saber (o no mejor que los responsables del contenido) en qué condiciones se hace. Es por ello, que el responsable operativo debe trasladar estas necesidades de negocio a las características del medio.

- Apoyar a otras unidades de negocio. Es posible que la unidad de los contenidos no se encargue de hacer todo el contenido del sitio. A veces, 


\section{Contexto Inmones s.}

unidades especializadas hacen su propio contenido. Por ejemplo, la página de ayuda para un determinado formulario o la información sobre un evento. En este caso, el responsable operativo debe ayudar, asistir y formar para que puedan hacer esto conforme a la estrategia.

\section{Los supervisores del contenido}

La supervisión del contenido tiene una consideración especial, para evitar dos situaciones poco deseables:

- Evitar contenido inapropiado. Es posible que en un momento dado alguien publique lo que no debe o cómo no debe. Internet tiene mala memoria, pero mucho impacto y un error puede tener un efecto muy negativo.

- Tener una cantidad de contenido ingobernable. Un sitio público puede tener miles de páginas. Si no revisamos el contenido recién hecho, es muy probable que este no se revise nunca por ingobernable. Eso no significa que un usuario no encuentre que el contenido esté mal o deje de estarlo (algo común en las webs de preguntas frecuentes).

Para hacer esta revisión es necesario contar con tres perfiles:

- Responsable operativo o persona delegada. Es quien comprueba que el contenido se adapta a la estrategia predefinida.

- Responsable de negocios. Es el propietario del contenido en concreto. Se asegura de que el contenido dice lo que tiene que decir y de la manera como contribuye a sus objetivos.

- Responsables web. Este perfil no tiene porqué actuar siempre, pero en los casos en los que un contenido se salga del cauce habitual (un tipo de imagen de un tamaño no previsto, un enlace a un tipo de documento nuevo, un cambio en los colores, etc.), debe asegurarse de que el efecto será el deseado.

\section{¿Qué más necesitamos?}

Existen una serie de competencias muy relacionadas con la gestión de contenidos, pero dependiendo de las características de la organización pueden estar diseminadas en otros departamentos. En ese caso, aunque no sea posible integrar estos conocimientos en la misma persona, sería importante colaborar con otros que tengan estas competencias para mejorar la creación de contenidos. Entre estos perfiles tenemos: 
- Analítica digital. La analítica ayuda a conocer qué contenido está funcionando y cuál no. Con ello aprendemos a conocer el público, el contenido y lo que más funciona.

- Experiencia de Usuario (UX). El contenido no es solo texto, es diseño, colores, imágenes, organización, etc. Tener a alguien con conocimientos de UX, es decir, la alineación de las características de las personas que usan la web con la navegación, la usabilidad y el diseño (Brightlabs, 2019), permite que el contenido logre lo que nos proponemos.

- Formación. La formación es crucial para orientar al personal de la organización en dos niveles. Por un lado, está la formación en la propia estrategia (dar a conocer qué hace y cómo actuar); por el otro, las cuestiones relacionadas con el contenido (escritura, diseño, maquetación si fuera necesario).

- Marketing y/o comunicación. Puede suceder que el responsable operativo esté más relacionado con el negocio que con la comunicación. Por ejemplo, que el contenido de la web de educación esté en el departamento de tecnología o de atención al ciudadano y no de comunicación. En ese caso, es muy importante contar con el apoyo de alguien del área de comunicación.

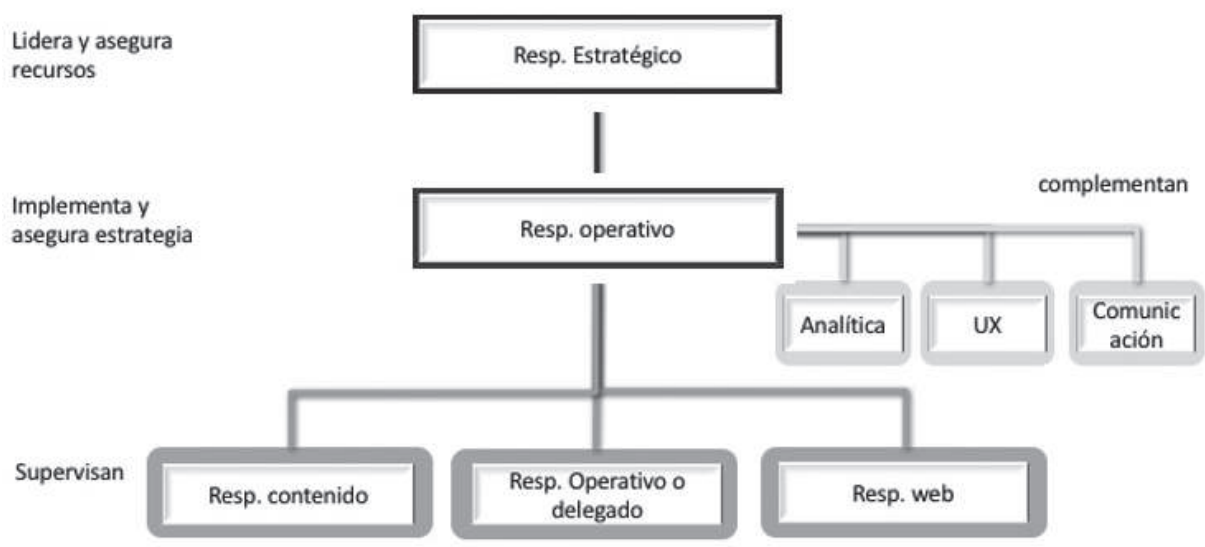

Figura 4. Gobernanza del contenido en entes locales.

Elaboración propia, a partir de Casey (2015).

Y con todo esto, tendríamos el esquema básico para poder garantizar que la estrategia funcione como se prevé. Estos son los términos ideales para la gestión de contenidos efectivos. De hecho, nos podemos encontrar con estructuras muy extensas cuya capacidad de control efectivo del contenido sea mínima. Sin embargo, la creación de contenido es efectiva, real, y, por lo general, adaptada mucho a las características de negocio y, en menor medida, a la estrategia general de la organización. 


\section{Conntexto unmones s.}

Cabe señalar que no todas las administraciones tienen estos medios, lo que genera, como hemos señalado anteriormente, un estrés o sobrecarga a los pocos responsables que generan esos contenidos. En esas circunstancias es necesario replantear una serie de cuestiones básicas:

- Establecer un contenido estático robusto. Vale la pena definir los bloques de contenido más importante; los cuales tiene que ser fijos: descripciones de servicios, infraestructuras, instrucciones generales y apartados de preguntas frecuentes. Son los que van a crear los cimientos básicos de la red.

- Utilizar un sistema de plantillas que reduzca el tiempo de creación de contenido (noticias, eventos, calendarios, actividades, etc.) para que la necesidad de creación de contenido sea la menos posible, generando una actualización mayor.

- Definir contenidos prioritarios para el público, tanto por contenido, como por frecuencia.

- Asumir un límite de actualización de contenido que se puede afrontar. No hay problema en que sea mucho o poco, el auténtico problema es pensar en llegar a objetivos que no son accesibles y que acaban generando frustración para el conjunto de la organización.

\section{Conclusiones}

A modo de conclusión podemos decir que el contenido es lo que acaba definiendo la naturaleza de una web municipal. La manera en la que articulamos lenguaje, terminología, formato y orden es lo que permite a dicho medio llegar a un tipo definido de público. Dicho de otra manera, más allá de los procesos impuestos (como la facturación electrónica), una web solo puede atrapar al público al que busca, y esto se hace a través del contenido.

En términos operativos, el contenido de una web municipal se puede agrupar, por su naturaleza, en tres planos:

- Servicios y trámites: la información que se refiere a lo que el ayuntamiento ofrece a la ciudadanía.

- Información institucional: lo referente a la composición, funcionamiento y estructura del gobierno, administración y política local.

- Información local: hace referencia a la vida y actualidad de la localidad más allá de la competencia pública.

Cada uno de estos elementos tiene requisitos y características diferenciadas que conciernen específicamente a la creación del contenido. Una persona ajena al gobierno local muy posiblemente deba pasar a lo largo de los tres 
tipos de contenido para poder llegar a conseguir sus objetivos. Uno de los retos fundamentales es la capacidad de ordenar y clasificar el contenido de manera que las personas puedan navegar de un tipo de contenido a otro, para conseguir el máximo de información que necesitan de manera voluntaria.

La generación de este contenido requiere, por lo tanto, la creación de estructuras que permitan alimentarlo y actualizarlo con los atributos necesarios para que sea eficaz. Esto va a depender de una serie de atributos estructurales, organizativos y de cantidad de objetos que se gestionan. La capacidad del ayuntamiento para poder organizar los recursos suficientes para abordar el contenido útil es la clave para hacer esta situación manejable. En todo caso, esa condición sobrevenida de tener que generar el contenido digital, además del resto de la actividad local, tiende a generar desequilibrios estructurales, bien sea hacia unos recursos insuficientes, incapaces de gestionar todas sus tareas, o bien hacia una generación excesiva e ingobernable de contenidos.

Para controlar esta situación es preciso avanzar hacia un modelo de gobernanza de contenidos que abarque las funciones de:

- Controlar el seguimiento de la estrategia de contenido.

- Supervisar que el contenido generado se adapte a las líneas de la organización.

- Generar el contenido específico en condiciones adaptadas al público.

- Definir los mensajes.

- Integrar todo ello de manera satisfactoria en la web.

Además de otros elementos complementarios.

Estas acciones son el itinerario crítico para una estrategia de contenidos eficaz. No hay manera de conseguir este resultado prescindiendo de estas funciones. Otra cuestión diferente es considerar si estas tareas las pueden hacer diversas personas, ya sea en el caso de organizaciones extensas o en el caso de ayuntamientos pequeños con poco personal. Cada uno de estos escenarios se encuentran con sus propios problemas y respuestas.

\section{Referencias}

Alba, C. (1997). Gobierno local y ciencia política: una aproximación. En C. Alba y F. Vanaclocha (Coords.), El sistema político local un nuevo escenario de gobierno (15-36). Madrid: Universidad Carlos III.

Bendickson, J., Muldoon, J., Liguori, E. \& Davis, P. (2016). Agency theory: the times, they are a-changin'. Management decision, 54(1), 174-193. 


\section{Contexto unmones.s.}

Brightlabs. (2019). Service Design - how it relates to CX and UX design. Recuperado en https://www.brightlabs.com.au/insights/service-designux-design-cx-design

Casey, M. (2015). The Content Strategy Toolkit: Methods, Guidelines, and Templates for Getting Content Right. United State of America: Copyrighted Material.

Echegaray, L., Urbano, I. y Barrutieta, G. (2017). Design Thinking, un modelo de aplicación para la Administración Pública. Madrid, España: Innap Investiga.

Eisenberg, J. \& Eisenberg, B. (2006). Call to Action: Secret Formulas to Improve Online Results. United State of America: Harper Collins.

Eisenberg, J., Eisenberg, B. \& Davis, L. (2002). Persuasive Online Copywriting: How to Take Your Words to the Bank. United State of America: Wizard Academy Press.

Kaushik, A. (2007). Web Analytics: An Hour a Day. United States of America: John Wiley and Sons.

Muñoz, G. y Elósegui, T. (2011). El arte de medir. Madrid, España: Profit.

Nielsen, J. (2000). Content Creation by Average People. Recuperado de https:// www.nngroup.com/articles/content-creation-for-average-people/

Solis, A. (2017). SEO. Claves esenciales. Madrid, España: Anaya.

Spool, J. (2008). Personas are NOT a document. Recuperado de https://archive. uie.com/brainsparks/2008/01/24/personas-are-not-a-document/

U.S. Government. (2016). Expectations and challenges: Informing the future of the Federal Front Door. Washington, EE UU: 18F, GSA, U.S. Government.

Vaughan, P. (2015). How to Create Detailed Buyer Personas for Your Business [Mensaje en un blog]. Recuperado de https://blog.hubspot.com/ marketing/buyer-persona-research

\section{Para citar este artículo:}

Jiménez, S. (2020). El contenido digital en las administraciones locales: condiciones ontológicas y organizativas para su creación y gestión. En-Contexto, 8(12), 93-114. 